\title{
Association between advanced interatrial block and small vessel diseases in the brain
}

\author{
Zhaolu Wang ${ }^{1}$, Huiyuan Qin ${ }^{2}$, Guilin Chen ${ }^{3}$, Vincent C. T. Mok $^{4}$, Yan Dai ${ }^{5}$, Yingyuan Cai ${ }^{6}, \mathrm{Xi} \mathrm{Cheng}^{6}$, \\ Yun Qian', Ming Chu ${ }^{2,7 \#}$, Xiaowei $\mathrm{Lu}^{6 \#}$ \\ ${ }^{1}$ Department of Neurology, ${ }^{2}$ Department of Cardiology, ${ }^{3}$ Department of Radiology, the First Affiliated Hospital of Nanjing Medical University, \\ Nanjing 210029, China; ${ }^{4}$ Department of Medicine and Therapeutics, Lui Che Woo Institute of Innovative Medicine, the Chinese University of \\ Hong Kong, Hong Kong, China; ${ }^{5}$ The First School of Clinical Medicine, ${ }^{6}$ Division of Neurology, Department of Geriatrics, the First Affiliated \\ Hospital of Nanjing Medical University, Nanjing 210029, China; ${ }^{7}$ Department of Cardiology, the Affiliated Hospital of Xuzhou Medical University, \\ Xuzhou 221006, China
}

"These authors contributed equally to this work.

Correspondence to: Xiaowei Lu, MD. Division of Neurology, Department of Geriatrics, the First Affiliated Hospital of Nanjing Medical University, Guangzhou Road 300\#, Nanjing 210029, China. Email: lxw@njmu.edu.cn; Ming Chu, MD. Department of Cardiology, the First Affiliated Hospital of Nanjing Medical University, Nanjing 210029, China. Email: chuming@njmu.edu.cn.

Background: The latest evidence shows the association of atrial cardiopathy with embolic strokes of undetermined source. Advanced interatrial block (aIAB) is an electrophysiological mark of atrial cardiopathy. This study investigated the relationship between $\mathrm{aIAB}$ and the burden of silent cerebral small vessel diseases (SVD) on magnetic resonance imaging in the absence of atrial fibrillation (AF) and atrial flutter.

Methods: This cross-sectional study included 499 patients with normal left ventricular ejection fraction (LVEF), who were free of AF, atrial flutter, stroke, and acute coronary syndrome in our hospital. aIAB was ascertained by digital electrocardiograms. Left atrial diameter, LVEF, and left ventricular posterior wall thickness (LVPWT) were measured on echocardiograms. Based on the presence of 4 manifestations of SVD, including white matter hyperintensity (WMH), lacunes, microbleeds, and enlarged perivascular spaces (EPVS) on magnetic resonance imaging, an ordinal SVD score (range, 0-4) was devised to reflect the total burden of cerebral SVD. The ordinal regression model was used to explore the association of aIAB with SVD burden after adjusting for confounding factors.

Results: The mean age was 67.7 years, and 327 (65.5\%) were male. A total of 23 (4.6\%) patients had aIAB. The number of patients with cerebral SVD scores of $0,1,2,3$, and 4 was 92 (18.4\%), 122 (24.4\%), 190 (38.1\%), 83 (16.6\%), and 12 (2.4\%), respectively. After adjusting for age, sex, hypertension, diabetes, hyperlipidemia, left atrial diameter, LVEF, and LVPWT, the regression model showed a significant association of aIAB with cerebral SVD score (OR =2.408, 95\% CI, 1.082-5.366).

Conclusions: Atrial cardiopathy indexed by aIAB was independently associated with a high burden of SVD in the brain.

Keywords: Advanced interatrial block (aIAB); atrial cardiopathy; small vessel diseases (SVD)

Submitted Nov 02, 2019. Accepted for publication Jan 18, 2020.

doi: 10.21037/qims.2020.02.02

View this article at: http://dx.doi.org/10.21037/qims.2020.02.02 


\section{Introduction}

Advanced interatrial block (aIAB) indicates the complete block of electrical stimulus conduction in the Bachmann's bundle region between the two atria (1). On electrocardiogram (ECG), aIAB is conveniently diagnosed by a $\mathrm{P}$-wave duration $>120 \mathrm{~ms}$, and a biphasic morphology of the P-wave in leads III, and aVF with biphasic morphology or notched morphology in lead II (2). Previous studies observed the association of aIAB with atrial remodeling [e.g., left atrium enlargement (LAE), atrial fibrosis, poor contractility] (3-5), and aIAB is thus recognized as a marker of atrial cardiopathy. In the general population, subjects with $\mathrm{aIAB}$ are more likely to develop atrial fibrillation (AF) compared with those without aIAB (incidence rate of AF: 29.8 vs. 6.8 per 1,000 person-years over a mean follow-up of 18.6 years, respectively) (6). In addition, O'Neal et al. found that aIAB is associated with incident ischemic stroke independent of $\mathrm{AF}$ over a follow-up of 22 years (7). Left atrial thrombosis may be one of the mechanisms explaining this phenomenon, and anticoagulation therapy among those with $\mathrm{aIAB}$ is under consideration but needs further investigation (8-10). Thus, aIAB may represent an early electrophysiological abnormality indicating prothrombotic remodeling of the left atrium (LA) (7).

Silent cerebral small vessel disease (SVD) refers to a group of pathological processes affecting the small arteries, arterioles, venules, and capillaries of the brain, which usually result in silent cerebral parenchyma damage detectable by magnetic resonance imaging (MRI), manifesting as white matter hyperintensity (WMH), enlarged perivascular spaces (EPVS), lacunes, and cerebral microbleeds (CMBs) (11). Hypoperfusion, blood-brain barrier alterations, dysfunction of the cerebral blood flow autoregulation, edema, apoptosis, inflammation, and genetic factors are presumed to contribute to the so-called ischemic forms of SVD (e.g., WMH), but this view is still controversial (11-13). A previous study showed that greater LA volumes and reduced LA reservoir function were associated with lacunes and $\mathrm{WMH}$, indicating the link between atrial dysfunction and SVD in the brain (14). However, aIAB is a separate entity from LA enlargement (4), and it remains unknown whether aIAB is associated with cerebral SVD.

In this study, we constructed an ordinal SVD score (range, 0-4) to reflect the burden of cerebral SVD, based on the presence of 4 manifestations of SVD (WMH, EPVS, lacunes, CMBs) on MRI. The association of aIAB with SVD burden was investigated in subjects with normal left ventricular ejection fraction (LVEF), who were free of AF, atrial flutter, stroke, and acute coronary syndrome, after adjusting for confounding factors [e.g., LA diameter (LAD), LVEF, and left ventricular posterior wall thickness (LVPWT)].

\section{Methods}

\section{Subjects}

We recruited consecutive stroke/acute coronary syndromefree subjects at the outpatient clinic of the Department of Geriatrics, the First Affiliated Hospital of Nanjing Medical University, between 2015 and 2016. These subjects were community citizens coming to our hospital for a health check-up. Resting digital electrocardiogram (ECG), echocardiography, and MRI were performed for all the patients. We excluded those with $\mathrm{AF}$ and atrial flutter. AF and atrial flutter were ascertained by ECG when recruited or from previous records of ECG (ECGs were performed annually for these health examination patients, and whenever they were previously hospitalized). Also, we only included patients with LVEF over 50\% (15). Ethics approval was obtained from the Nanjing Medical University, and written informed consent was obtained from each participant. All methods were performed following the relevant guidelines and regulations (the Declaration of Helsinki and the Nanjing Medical University policies).

\section{Demography and vascular risk factors}

We collected data on basic demography (age and sex) and vascular risk factors. Hypertension was defined as systolic blood pressure $>140 \mathrm{mmHg}$ and/or diastolic blood pressure $>90 \mathrm{mmHg}$, a history of hypertension, or use of antihypertensive medication. Diabetes mellitus (DM) was defined as fasting serum glucose $\geq 7.0 \mathrm{mmol} / \mathrm{L}$, postprandial serum glucose $\geq 11.1 \mathrm{mmol} / \mathrm{L}$, or the use of hypoglycemic agents or insulin. Hyperlipidemia was defined as a total cholesterol level of $\geq 5.2 \mathrm{mmol} / \mathrm{L}$, a low-density lipoprotein cholesterol level of $\geq 2.6 \mathrm{mmol} / \mathrm{L}$, a triglyceride level of $\geq 1.70 \mathrm{mmol} / \mathrm{L}$, or the use of lipid-lowering drugs.

\section{ECG and echocardiography}

Resting digital ECG was performed to detect aIAB, which was defined as a $\mathrm{P}$-wave duration $>120 \mathrm{~ms}$ and a 
biphasic morphology of the P-wave in leads III and aVF with biphasic morphology or notched morphology in lead II (2). LAD, LVEF, and LVPWT were recorded on echocardiography (16). LAD measurement was performed during end ventricular systole. These data were rated by a cardiologist blinded to the clinical information of participants.

\section{Neuroimaging examinations of SVD}

Brain MRI (3.0 Tesla, TIM Trio, Simens, Erlangen, Germany) was performed to detect SVD. All patients received T1-weighted imaging, T2-weighted imaging, fluid-attenuated inversion recovery (FLAIR), and sensitivity-weighted imaging (SWI) using standard protocols. Deep and periventricular WMH were rated using the Fazekas scale from 0 to 3 on FLAIR images (17). Lacunes were identified as a round or ovoid hypointense lesions on FLAIR (with hyperintense rim) and T1-weighted images of between 3 and $15 \mathrm{~mm}$ in diameter (18). Cerebral microbleeds, defined as focal areas of a very low signal intensity of $10 \mathrm{~mm}$ maximum in diameter, were counted on SWI (19). EPVS was defined as small, sharply delineated structures of cerebrospinal fluid intensity on imaging that followed the orientation of the perforating vessels and ran perpendicular to the brain surface. They were of high signal on T2 and low signal on T1 and fluid-attenuated inversion recovery sequences (18). EPVS was rated in the basal ganglia and centrum semiovale separately, according to a previously validated semiquantitative scale (Grade 0-4) (20). EPVS was counted on one side of the brain that had more EPVS compared with the other side. All ratings were performed blind to patients' clinical data. Both intrarater agreements and interrater agreements between 2 raters were good or excellent for rating these neuroimaging markers on 30 randomly selected patients (the intraclass correlation coefficient ranged from 0.81 to 0.99 ). The presence of lacunes or CMBs was defined as $\geq 1$ lacune or $\geq 1$ CMBs, respectively. The presence of WMH was defined as either (early) confluent deep WMH (Fazekas score 2 or 3) or irregular periventricular WMH extending into the deep white matter (Fazekas score 3). The presence of EPVS was defined as a Grade 2-4 of EPVS (21). One point was awarded for each of the above 4 neuroimaging presences, constructing an ordinal score ranging from 0 to 4 to reflect the total burden of cerebral SVD (21). The EPVS in basal ganglia, rather than centrum semiovale, was involved in this SVD score because EPVS in basal ganglia are specifically associated with cerebral SVD $(21,22)$.

\section{Statistics}

Continuous and categorical variables were reported as means (standard deviations) and frequencies (percentages), respectively. Comparisons between groups were conducted using Student's $t$-test, Mann-Whitney $U$ test, Chi-square, or Fisher's exact test, as appropriate. Ordinal regression was used to investigate the association of aIAB with the burden of cerebral SVD, adjusting for age, sex, hypertension, DM, hyperlipidemia, LAD, LVEF, and LVPWT. SPSS 22.0 was used for analysis, and $\alpha$ was set at 0.05 for all statistical analyses.

\section{Results}

A total of 499 patients were included in this study. The mean age was 67.7 years (SD 13.6), and 327 (65.5\%) were male. aIAB were detected in $23(4.6 \%)$ patients. WMH, EPVS in BG, EPVS in centrum semiovale, lacunes, and CMBs were present in 310 (62.1\%), $334(66.9 \%), 394$ (79.0\%), 134 (26.9\%), and 21 (4.2\%) patients, respectively. The number of patients with a cerebral SVD score of 0,1 , 2, 3, and 4 was 92 (18.4\%), 122 (24.4\%), 190 (38.1\%), 83 (16.6\%), and $12(2.4 \%)$, respectively.

Compared with subjects without aIAB (Table 1), those with $\mathrm{aIAB}$ had a larger $\mathrm{LAD}(38.3$ vs. $35.1 \mathrm{~mm}, \mathrm{P}=0.001$ ), lower LVEF (63.1 vs. 65.1, $\mathrm{P}=0.007$ ), and a higher incidence of $\mathrm{WMH}(82.6 \%$ vs. $61.1 \%, \mathrm{P}=0.038)$. Chi-square also showed a significant association of aIAB with SVD total score $(\mathrm{P}=0.024$; Table 1$)$.

The association of aIAB with SVD burden is shown in Table 2. An ordinal regression model found that aIAB was associated with a high SVD score $(\mathrm{OR}=2.408,95 \%$ CI, 1.082-5.366, P=0.031), after adjusting for age, sex, hypertension, diabetes, hyperlipidemia, LAD, LVEF, and LVPWT. In addition, age (OR $=1.058,95 \%$ CI, $1.044-$ $1.073, \mathrm{P}<0.001)$, hypertension $(\mathrm{OR}=1.824,95 \% \mathrm{CI}, 1.250$ 2.662, $\mathrm{P}=0.002)$, LVEF (OR $=0.899,95 \% \mathrm{CI}, 0.856-0.946$, $\mathrm{P}<0.001)$, and LVPWT (OR $=1.363,95 \%$ CI, 1.188-1.565, $\mathrm{P}<0.001)$ were all related to a high burden of cerebral SVD.

\section{Discussion}

This cross-sectional study observed an association of aIAB with the total burden of SVD in the brain, independent of LAD, LVEF, LVPWT, and other possible confounding 
Table 1 Characteristics of the patients with and without aIAB

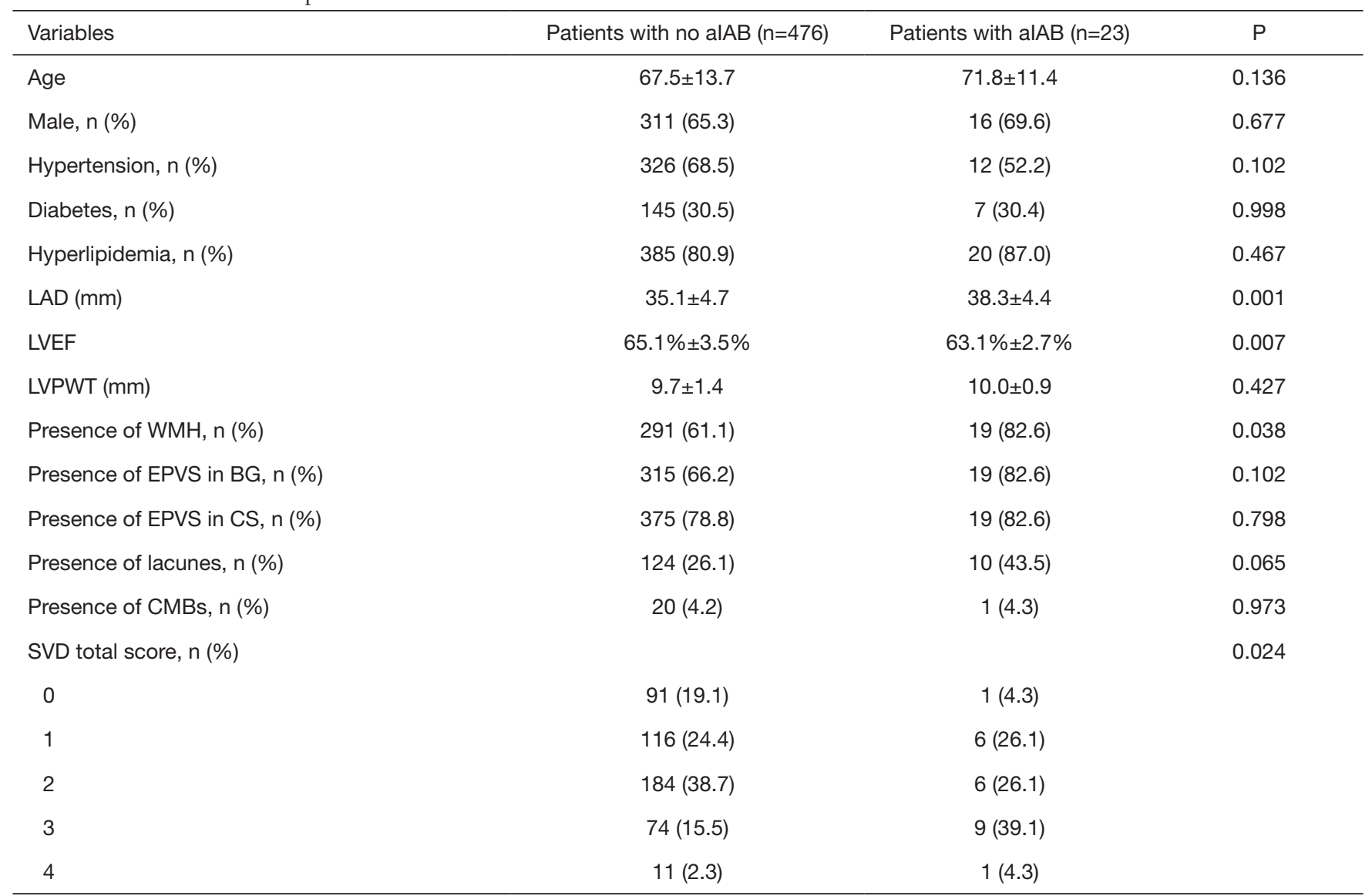

Continuous and categorical variables are presented as mean $\pm \mathrm{SD}$ and percentages, respectively. alAB, advanced interatrial block; $B G$, basal ganglia; CS, centrum semiovale; CMBs, cerebral microbleeds; EPVS, enlarged periventricular spaces; LAD, left atrial diameter; LVEF, left ventricular ejection fraction; LVPWT, left ventricular posterior wall thickness; SVD, small vessel diseases; WMH, white matter hyperintensities.

Table 2 Associations of aIAB with total SVD score in multivariate ordinal regression analysis

\begin{tabular}{lcc}
\hline Variables & OR $(95 \% \mathrm{Cl})$ & $\mathrm{P}$ \\
\hline Age & $1.058(1.044-1.073)$ & $<0.001$ \\
Male & $0.748(0.525-1.064)$ & 0.106 \\
Hypertension & $1.824(1.250-2.662)$ & 0.002 \\
Diabetes & $1.156(0.804-1.662)$ & 0.433 \\
Hyperlipidemia & $1.358(0.870-2.117)$ & 0.178 \\
LAD $(\mathrm{mm})$ & $0.980(0.945-1.018)$ & 0.306 \\
LVEF & $0.899(0.856-0.946)$ & $<0.001$ \\
LVPWT (mm) & $1.363(1.188-1.565)$ & $<0.001$ \\
alAB & $2.408(1.082-5.366)$ & 0.031
\end{tabular}

alAB, advanced interatrial block; SVD, small vessel diseases; LAD, left atrial diameter; LVEF, left ventricular ejection fraction; LVPWT, left ventricular posterior wall thickness. 
factors. To the best of our knowledge, this is the first study to investigate the relationship between $\mathrm{aIAB}$ and cerebral SVD.

aIAB is a blockade of the electrical impulse between the right and left atrium. It is easily recognized on resting ECG and is related to LAE, atrial fibrosis, poor atrial contractility, high incidence of $\mathrm{AF}$, ischemic stroke, and all-cause mortality after ischemic stroke $(3-7,23)$. Therefore, it is a marker of atrial cardiopathy, which is a term to describe structural or electrophysiological changes affecting the atria that potentially causes clinically relevant manifestations (24). aIAB is not uncommon in the general population (around 1\%) (6), and several small studies reported a higher prevalence of aIAB in subjects with heart failure (10-23.5\%) $(1,25)$ or AF $(39 \%)(26)$. In our study, the prevalence of aIAB was $4.6 \%$, and patients with $\mathrm{aIAB}$ had a bigger LAD compared to patients without aIAB in univariable analysis (Table 1). aIAB, although often accompanied by LA enlargement, is indeed a separate entity from LA enlargement (4), and it may precede or occur without LA enlargement and atrial arrhythmia (9). The association of atrial cardiopathy indexed by aIAB with cerebral SVD burden in this study is independent of LAD, LVEF, and LVPWT, indicating that LA electrophysiological dysfunction is independent of these cardiac structural/ functional abnormalities in this cardiac-brain link.

Consistent with our study, a latest population-based study observed an association of atrial cardiopathy (defined as excessive supraventricular ectopy on 24-hour Holter ECG with WMH (27). The exact mechanism for the association of aIAB with cerebral SVD is unclear, but may be accounted for by the following phenomena: first, aIAB may represent a hypercoagulable and prothrombotic state of LA (7). Previous cohort studies observed the association of $\mathrm{aIAB}$ with an increased risk of ischemic stroke, independent of atrial arrhythmia $(7,8)$. aIAB-induced electromechanical dysfunction leads to the sluggishly contractile left atrium, which may form a nidus for microthrombi (28), causing SVD in the brain (e.g., WMH and lacunes). Thus, prophylactic anticoagulation in $\mathrm{IAB}$ without associated atrial tachyarrhythmias is under consideration (9), but it still needs further exploration in prospective studies, especially randomized controlled trials. Second, atrial cardiopathy reflected by aIAB may compromise the perfusion to the brain, causing cerebral SVD. A cross-sectional study found that reduced LA reservoir function was associated with a higher burden of $\mathrm{WMH}$ and silent brain infarcts in a stroke-free community-based cohort (14), suggesting the contribution of chronic cardiogenic hypoperfusion to SVD in the brain. Third, the relationship reported here might indicate the presence of shared risk factors between atrial cardiopathy and cerebral SVD, although the risk factors were adjusted.

This study has several strengths. First, we constructed an SVD score to represent the burden of cerebral SVD, which could better capture the overall effect of SVD than a single MRI manifestation of cerebral SVD. Second, the large sample size makes it possible to adjust for confounding factors, including LAD, LVEF, and LVPWT, which are structural or functional cardiac changes.

Our study also has limitations. First, participants did not undergo continuous heart-rhythm monitor to detect occult $\mathrm{AF}$ or atrial flutter, and we cannot exclude the possibility that subclinical AF mediated the association of aIAB with silent cerebral SVD. However, AF and atrial flutter were ascertained by ECG when recruited or from previous records of ECG. Participants in this study received 3-5 ECGs before they were recruited according to their medical records. Multiple ECG monitoring decreases the possibility of false-negative AF/atrial flutter. In addition, we reviewed the medical records of these 23 participants with $\mathrm{aIAB}$ and performed 24-hour Holter ECG monitor on those who had not undergone Holter monitoring previously. Among them, 10 had already undergone a 24-hour Holter ECG monitoring, 12 accepted the 24-hour Holter monitoring during the study, and 1 patient was lost to follow-up with no Holter monitoring available. Only 1 patient presented with paroxysmal $\mathrm{AF}$ among these 22 participants. The association of aIAB with silent SVD still held (data not shown) after excluding those 2 patients ( $1 \mathrm{AF}$ patient and the 1 patient lost to follow-up now). This association would be expected to be more significant if occult AF carriers were also excluded among participants without aIAB, supposing AF was a confounding factor. Furthermore, even if undetected occult $\mathrm{AF}$ mediates some of the associations of $\mathrm{aIAB}$ with silent SVD, this finding on standard ECG screening is easier and more cost-effective than the continuous heart-rhythm monitor. Nevertheless, future studies with continuous heart-rhythm monitoring are needed. Our second limitation is the observational cross-sectional design of this study, which cannot confirm a causal effect between atrial cardiopathy and cerebral SVD. Third, despite several "events" (the presence of SVD manifestations on MRI), there are still few patients with aIAB. Overadjustment may be a concern in the statistical analysis. Fourth, the older age in this study limited its generalizability to all populations. 
In conclusion, this cross-sectional study demonstrated an association of $\mathrm{AIAB}$ with a high burden of cerebral SVD. aIAB might help to improve risk stratification of individuals at an elevated risk of subclinical cerebrovascular diseases. Prospective studies with larger sample sizes are needed to explore the mechanisms behind this.

\section{Acknowledgments}

Funding: This work was supported by the National Natural Science Foundation of China (Grant No. 81771286), the Cadre Health Research Project of Jiangsu Provincial Commission of Health and Family Planning (BJ17019) and the Shuangchuang Plan of Jiangsu Province [2016].

\section{Footnote}

Conflicts of Interest: The authors have no conflicts of interest to declare.

Ethical Statement: Ethics approval was obtained from the Nanjing Medical University, and written informed consent was obtained from each participant.

Open Access Statement: This is an Open Access article distributed in accordance with the Creative Commons Attribution-NonCommercial-NoDerivs 4.0 International License (CC BY-NC-ND 4.0), which permits the noncommercial replication and distribution of the article with the strict proviso that no changes or edits are made and the original work is properly cited (including links to both the formal publication through the relevant DOI and the license). See: https://creativecommons.org/licenses/by-nc-nd/4.0/.

\section{References}

1. de Luna AB, Masso-van Roessel A, Robledo LAE. The Diagnosis and Clinical Implications of Interatrial Block. Eur Cardiol 2015;10:54-9.

2. Maheshwari A, Norby FL, Roetker NS, Soliman EZ, Koene RJ, Rooney MR, O'Neal WT, Shah AM, Claggett BL, Solomon SD, Alonso A, Gottesman RF, Heckbert SR, Chen LY. Refining Prediction of Atrial FibrillationRelated Stroke Using the P2-CHA2DS2-VASc Score. Circulation 2019;139:180-91.

3. Lacalzada-Almeida J, Izquierdo-Gomez MM, BelleyoBelkasem C, Barrio-Martinez P, Garcia-Niebla J, Elosua R, Jimenez-Sosa A, Escobar-Robledo LA, Bayes de Luna
A. Interatrial block and atrial remodeling assessed using speckle tracking echocardiography. BMC Cardiovasc Disord 2018;18:38.

4. Bayes de Luna A, Platonov P, Cosio FG, Cygankiewicz I, Pastore C, Baranowski R, Bayes-Genis A, Guindo J, Vinolas X, Garcia-Niebla J, Barbosa R, Stern S, Spodick D. Interatrial blocks. A separate entity from left atrial enlargement: a consensus report. J Electrocardiol 2012;45:445-51.

5. Benito EM, De Luna AB, Baranchuk A, Mont L. Extensive atrial fibrosis assessed by late gadolinium enhancement cardiovascular magnetic resonance associated with advanced interatrial block electrocardiogram pattern. Europace 2017;19:377.

6. O'Neal WT, Zhang ZM, Loehr LR, Chen LY, Alonso A, Soliman EZ. Electrocardiographic Advanced Interatrial Block and Atrial Fibrillation Risk in the General Population. Am J Cardiol 2016;117:1755-9.

7. O'Neal WT, Kamel H, Zhang ZM, Chen LY, Alonso A, Soliman EZ. Advanced interatrial block and ischemic stroke: The Atherosclerosis Risk in Communities Study. Neurology 2016;87:352-6.

8. Hughes TM, Worrall BB. Acute interatrial block is a distinct risk factor for ischemic stroke. Neurology 2016;87:344-5.

9. Chhabra L, Gowdar S. Interatrial Block to Guide the Thromboembolic Prevention Strategy: Should It be the Next Step? Am J Cardiol 2017;120:e7.

10. Martínez-Sellés M, Baranchuk A, Elosua R, de Luna AB. Rationale and design of the BAYES (Interatrial Block and Yearly Events) registry. Clin Cardiol 2017;40:196-9.

11. Pantoni L. Cerebral small vessel disease: from pathogenesis and clinical characteristics to therapeutic challenges. Lancet Neurol 2010;9:689-701.

12. Joutel A, Chabriat H. Pathogenesis of white matter changes in cerebral small vessel diseases: beyond vesselintrinsic mechanisms. Clin Sci (Lond) 2017;131:635-51.

13. Humayun $\mathrm{H}$, Yao J. Imaging the aged brain: pertinence and methods. Quant Imaging Med Surg 2019;9:842-57.

14. Russo C, Jin Z, Liu R, Iwata S, Tugcu A, Yoshita M, Homma S, Elkind MS, Rundek T, Decarli C, Wright CB, Sacco RL, Di Tullio MR. LA volumes and reservoir function are associated with subclinical cerebrovascular disease: the CABL (Cardiovascular Abnormalities and Brain Lesions) study. JACC Cardiovasc Imaging 2013;6:313-23.

15. Oeing CU, Tschope C, Pieske B. The new ESC Guidelines for acute and chronic heart failure 2016. Herz 
2016;41:655-63.

16. Kovács Z, Kormanyos A, Domsik P, Kalapos A, Lengyel C, Ambrus N, Ajtay Z, Piros GA, Forster T, Nemes A. Left ventricular longitudinal strain is associated with mitral annular fractional area change in healthy subjectsResults from the three-dimensional speckle tracking echocardiographic MAGYAR-Healthy Study. Quant Imaging Med Surg 2019;9:304-11.

17. Fazekas F, Kleinert R, Offenbacher H, Schmidt R, Kleinert G, Payer F, Radner H, Lechner H. Pathologic correlates of incidental MRI white matter signal hyperintensities. Neurology 1993;43:1683-9.

18. Wardlaw JM, Smith EE, Biessels GJ, Cordonnier C, Fazekas F, Frayne R, Lindley RI, O'Brien JT, Barkhof F, Benavente OR, Black SE, Brayne C, Breteler M, Chabriat H, Decarli C, de Leeuw FE, Doubal F, Duering M, Fox NC, Greenberg S, Hachinski V, Kilimann I, Mok V, Oostenbrugge R, Pantoni L, Speck O, Stephan BC, Teipel S, Viswanathan A, Werring D, Chen C, Smith C, van Buchem M, Norrving B, Gorelick PB, Dichgans $M$. Neuroimaging standards for research into small vessel disease and its contribution to ageing and neurodegeneration. Lancet Neurol 2013;12:822-38.

19. Greenberg SM, Vernooij MW, Cordonnier C, Viswanathan A, Al-Shahi Salman R, Warach S, Launer LJ, Van Buchem MA, Breteler MM. Cerebral microbleeds: a guide to detection and interpretation. Lancet Neurol 2009;8:165-74.

20. Doubal FN, MacLullich AM, Ferguson KJ, Dennis MS, Wardlaw JM. Enlarged perivascular spaces on MRI are a feature of cerebral small vessel disease. Stroke 2010;41:450-4.

21. Staals J, Makin SD, Doubal FN, Dennis MS, Wardlaw JM. Stroke subtype, vascular risk factors, and total MRI brain small-vessel disease burden. Neurology 2014;83:1228-34.

22. Klarenbeek P, van Oostenbrugge RJ, Rouhl RP, Knottnerus IL, Staals J. Ambulatory blood pressure in patients with lacunar stroke: association with total MRI burden of

Cite this article as: Wang Z, Qin H, Chen G, Mok VCT, Dai Y, Cai Y, Cheng X, Qian Y, Chu M, Lu X. Association between advanced interatrial block and small vessel diseases in the brain. Quant Imaging Med Surg 2020;10(3):585-591. doi: 10.21037/ qims.2020.02.02 cerebral small vessel disease. Stroke 2013;44:2995-9.

23. Baturova MA, Lindgren A, Shubik YV, Carlson J, Platonov PG. Interatrial block in prediction of all-cause mortality after first-ever ischemic stroke. BMC Cardiovasc Disord 2019;19:37.

24. Goette A, Kalman JM, Aguinaga L, Akar J, Cabrera JA, Chen SA, Chugh SS, Corradi D, D'Avila A, Dobrev D, Fenelon G, Gonzalez M, Hatem SN, Helm R, Hindricks G, Ho SY, Hoit B, Jalife J, Kim YH, Lip GY, Ma CS, Marcus GM, Murray K, Nogami A, Sanders P, Uribe W, Van Wagoner DR, Nattel S. EHRA/HRS/APHRS/ SOLAECE expert consensus on atrial cardiomyopathies: Definition, characterization, and clinical implication. Heart Rhythm 2017;14:e3-40.

25. Escobar-Robledo LA, Bayes-de-Luna A, Lupon J, Baranchuk A, Moliner P, Martinez-Selles M, Zamora E, de Antonio M, Domingo M, Cediel G, Nunez J, SantiagoVacas E, Bayes-Genis A. Advanced interatrial block predicts new-onset atrial fibrillation and ischemic stroke in patients with heart failure: The "Bayes' Syndrome-HF" study. Int J Cardiol 2018;271:174-80.

26. Gul EE, Pal R, Caldwell J, Boles U, Hopman W, Glover B, Michael KA, Redfearn D, Simpson C, Abdollah H, Baranchuk A. Interatrial block and interatrial septal thickness in patients with paroxysmal atrial fibrillation undergoing catheter ablation: Long-term follow-up study. Ann Noninvasive Electrocardiol 2017. doi: 10.1111/ anec. 12428 .

27. Hisamatsu T, Miura K, Fujiyoshi A, Kunimura A, Ito T, Miyazawa I, Torii S, Shiino A, Nozaki K, Kanda H, Arima H, Ohkubo T, Ueshima H. Association between excessive supraventricular ectopy and subclinical cerebrovascular disease: a population-based study. Eur J Neurol 2019;26:1219-25.

28. Goyal SB, Spodick DH. Electromechanical dysfunction of the left atrium associated with interatrial block. Am Heart J 2001;142:823-7. 